\title{
Virtual screening of piperine derivatives with potential anti- leishmanial activity
}

Natália F. de Sousa (PG) ${ }^{1}$, Pollyana C. G. e Silva (PG) ${ }^{1}$, Helivaldo D. S. Souza (PQ) ${ }^{2}$, Normando A. S. Costa (PQ) ${ }^{1}$, Marcus T. Scotti (PQ) ${ }^{1}$, Luciana Scotti1 (PQ) ${ }^{1}$, Petrônio F. Athayde-Filho (PQ) ${ }^{2}$, José M. Barbosa-Filho (PQ) ${ }^{1}$

${ }^{1}$ Programa de Pós-Graduação em Produtos Naturais e Sintéticos Bioativos, Universidade Federal da Paraíba, João Pessoa - Paraíba Brasil; ${ }^{2}$ Departamento de Química, Universidade Federal da Paraíba, João Pessoa - Paraíba - Brasil.. nferreiradesousa.nfs@gmail.com *

\begin{tabular}{|l|l|} 
Graphical Abstract \\
Introduction: Piperine is a natural alkaloid found in Piper \\
nigrum. This work aims to perform a virtual screening of \\
20 synthetic piperine derivatives with potential anti- \\
leishmanial activity. Methodology: A classificatory \\
prediction model concerning the Leishmania infantum \\
Promastigote cell form in Knime software was built. The \\
compounds were subjected to Molecular Docking in the \\
Molegro Virtual Docker v.6.0.1 software, using the CYP51 \\
target complexed to the inhibitor Fluconazole obtained \\
from the Protein Data Bank. The molecules under study \\
were submitted to a consensus calculation involving the \\
activity probability obtained in the elaborated model, as \\
well as in the molecular docking simulation performed. \\
Results: The prediction model was more than 78\% accurate \\
in the test and in the cross-validation, selecting the 20 \\
synthetic derivatives under study as potentially active for \\
the promastigote form. In Docking, only 9 of the molecules \\
of the 20 molecules selected by the model had better energy \\
than the ligand. In the consensus calculation, nine \\
compounds had a probability above 50\%. Among the \\
structures, molecule 20 was considered the one with the \\
best performance in the study developed.Conclusions: The \\
virtual screening performed was able to identify the \\
compounds with the highest probability of activity. \\
Palavras-Chave: Piperina; Estudo computacional; \\
Leishmaniose.
\end{tabular}




\section{Introduction}

Leishmaniasis is characterized by being an infectious pathology caused by protozoa of the genus Leishmania sp belonging to family Trypanosomatidae ${ }^{[1,2]}$. Transmission to humans occurs through the bite of infected female sand flies [3].

Among the species of Leishmania existing, the Leishmania infantum is characterized by being a flagellate protozoan that causes Visceral Leishmaniasis in the Americas [4]. This disease is known as kala azar, tropical splenomegaly and dundum fever and is characterized as a systemic infectious disease that presents as symptoms of long-term fever, enlarged liver and spleen, weight loss, weakness, reduced muscle strength and anemia [5].

Existing compounds for treatment have drawbacks that demonstrate the need to discover new therapeutic agents for this purpose. Among the barriers associated with the therapy currently adopted are the diversity of side effects, high levels of toxicity and cases of resistance by the parasite [6,7].

Piperine is a natural alkaloid found in Piper nigrum, serves as a basis for obtaining new molecules that have a range of biological activities, mainly leishmania [8].

One of the available ways to discover new drugs is to carry out studies of the Quantitative Relationship between Chemical Structure and Biological Activity (QSAR), which uses information about compounds with known activity values to build predictive models, as well as to perform chemical similarity of search or based on receiver structure $[9,10]$.

This work aims to perform a virtual screening of 20 synthetic piperine derivatives with potential anti-leishmanial activity.

\section{Materials and Methods}

\section{Database}

The elaborated prediction model was based on the classification of the data, using nominal variables and was constructed from two sets of structures.

The first set was obtained from the ChEMBL database $[11,12]$ and was composed of 290 structures with activity potential against Leishmania infantum Promastigote Form.

The compounds were classified according to the pIC50 value (- $\log$ of IC50 (mol / L), with 134 active (pIC50 $\geq 4.84$ ) and 131 inactive (pIC50 <4.84). It is worth mentioning that IC50 is the concentration required to inhibit $50 \%$ of Leishmania infantum Promastigote Form activity.

The prediction set consists of unpublished 20 piperine derivatives with potential for synthesis.

\section{Standardization of Chemical Structures}

The chemical structures were converted into SMILES, these being the input data for Marvin [13]. Standardizer software [14] was used to standardize the chemical structures, with the addition of hydrogen atoms $(\mathrm{H})$, aromatic ring and 3D structure generation.

\section{Descriptors}


The biological and physicochemical properties of the molecules were generated by the VolSurf software (Volume and Surface) [15,16], which encode different spatial and geometric dimensions and are generated from the 3D structure.

\section{Statistical Analyzes}

The model was generated by the statistical software KNIME 3.1.0 [17,18], using Random Forest (RF) as calculation algorithm. (RF) is a supervised algorithm that is based on the combination of prediction trees, so that each tree depends on the values of a randomly sampled vector and the same distribution for all the trees in the forest.

\section{Molecular Docking}

To carry out the Molecular Docking simulations, the search for the target protein and the respective ligand of the Leishmania species under study was performed. For L. infantum the structure of the receptors used in the study were: Sterol 14-alpha demethylase - CYP51 (PDB: 3L4D) [19].

Proteins were obtained in the Protein Data Bank (PDB) library (https://www.rcsb.org/) [20]. The choice of targets was carried out through literature review on the mechanism of action involved in the inhibition of the studied parasites, as well as taking into account the structural similarity of the compounds under study with the ligands of the respective enzymes.

Before performing the Molecular Docking simulation, the Redocking step was performed, in order to validate the Docking performed later. Both procedures were performed using the Molegro Virtual Docker (MVD) v.6.0.1 software [21].

\section{Calculation of Combined Probability}

It is performed to assess the potential of multitarget molecules through docking, combined with the virtual screening model. In general, it aims to select molecules that are potentially active for several enzymes that were also predicted to be active in the created virtual screening model. For this type of calculation, the following formula is used:

$$
\operatorname{Prob} \operatorname{Comb}=\frac{(\text { Prob }+ \text { Cross }) x A p}{2+\text { Cross }}, \text { If Prob Comb }>0,5
$$

Where, Prob is the probability of active potential, Cross is the Cross Validation value of the virtual screening model and Ap is the predicted value in the model of the activity of each molecule. and this combined probability is conditioned, that is, only molecules that present values above 0.5 will be considered potentially active. Combined probability values were calculated for the 24 molecules under study [22,23].

\section{Results and Discussion (optional)}

\section{Rating Rate}

The classification rate of the model was evaluated by the receiver operating characteristic (ROC) graph, corresponding in the test set to 0.90 , in Cross Validation obtained a value of 0.91 . It should be 
noted that a perfect model presents area under the curve equal to 1, in this way, it is possible to state that the model is capable of performing a high classification rate for the RF method.

\section{Prediction Assessment}

The Matthews Correlation Coefficient (MCC) was used to evaluate the prediction of the model, resulting in 0.61 for the test set and 0.60 for Cross Validation, respectively, indicating that the model has a good prediction.

\section{Activity Probability}

By means of the probability, the elaborated model was used to triage the possible activity of Piperine derivatives against Leishmania infantum. The molecules that reached a probability of being active greater than $50 \%$, totaled the 20 molecules components of the prediction series, 7 with probability of activity between 50 and 59\%; 11 with activity probability between 60 and 69\%; 1 molecules with probability above $70 \%$. Recalling that the applicability domain was reliable for 20 molecules, thus being only one molecule component of the prediction series classified as unreliable.

\section{Molecular Docking}

No Docking, o ligante Fluconazol apresentou energia -117,578 kcal|mol. Apenas 9 das moléculas selecionadas pelo modelo obtiveram energia melhor que o ligante, situando-se na faixa de $-118,128$ a $127,316 \mathrm{kcal} \mid \mathrm{mol}$. As poses mais estáveis corresponderam respectivamente aos compostos 6, 3, 8, 15, $13,19,20,11$ e 14.

\section{Calculation of Combined Probability}

In the consensus calculation, nine compounds had a probability above 50\%, respectively 13 (68\%); 14 (69\%); 6 and 8 (71\%); 15 (72\%); 3 (74\%); $11(76 \%) ; 19(77 \%)$ and $20(78 \%)$. Among the structures, molecule 20 was considered the one with the best performance in the study developed.

\section{Conclusions}

The virtual screening performed was able to identify the compounds with the highest probability of activity.

\section{References}

1. Catorze, M.G.B. Leishmaniose e SIDA. Med. Cutan. Ibero. Lat. Am. 2005, 33, 237-250.

2. Paternina-Gómez, M.; Díaz-Olmos, Y.; Paternina, L.E.; Bejarano, E.E. High prevalence of infection with Leishmania (Kinetoplastea: Trypanosomatidae) in dogs in northern Colombia. Biomédica 2013, 33, 375-382.

3. Cochero, S.; Anaya, Y.; Díaz, Y.; Paternina, M.; Luna, A.; Paternina, L.; Bejarano, E.E. Infección natural de Lutzomyia cayennensis cayennensis con parásitos tripanosomatídeos (Kinetoplastida: Trypanosomatidae) en Los Montes de María, Colombia. Rev. Cubana Med. Trop. 2007, 59, 0.

4. Marcondes, M.; Rossi, C.N. Leishmaniose visceral no Brasil. Brazilian J. Vet. Res. Anim. Sci. 2013, 50, $341-352$.

5. Secretaria de VigiLância em Saúde Vigilância em saúde no Brasil 2013-2019. Bol. epidemiológico 2019 , 156.

6. Uliana, S.R.B.; Trinconi, C.T.; Coelho, A.C. Chemotherapy of leishmaniasis: present challenges. Parasitology 2018, 145, 464-480.

7. Silva, D.G.; Gillespie, J.R.; Ranade, R.M.; Herbst, Z.M.; Nguyen, U.T.T.; Buckner, F.S.; Montanari, C.A.; Gelb, M.H. New class of antitrypanosomal agents based on imidazopyridines. ACS Med. Chem. Lett. 2017, 8, 766-770.

8. Yasir, A.; Ishtiaq, S.; Jahangir, M.; Ajaib, M.; Salar, U.; Khan, K.M. Biology-oriented synthesis (BIOS) of piperine derivatives and their comparative analgesic and antiinflammatory activities. Med. Chem. (Los. Angeles). 2018, 14, 269-280.

9. Alves, V.M.; Braga, R.C.; Muratov, E.N.; Horta, C. Quim. Nova,. 2018, 41, 202-212.

10. Gasteiger, J. Chemoinformatics: Achievements and challenges, a personal view. Molecules $2016,21$.

11. EMBL-EBI ChEMBL Data Base 2019.

12. Gaulton, A.; Bellis, L.J.; Bento, A.P.; Chambers, J.; Davies, M.; Hersey, A.; Light, Y.; McGlinchey, S.; Michalovich, 
D.; Al-Lazikani, B.; et al. ChEMBL: A large-scale bioactivity database for drug discovery. Nucleic Acids Res. 2012, 40, 1100-1107.

13. ChemAxon Marvin Sketch.

14. ChemAxon Standardizer.

15. Cruciani, G.; Pastor, M.; Guba, W. VolSurf: A new tool for the pharmacokinetic optimization of lead compounds. Eur. J. Pharm. Sci. 2000, 11.

16. Molecular Discovery Volsurf Program V 1.7.0.

17. Knime.

18. Berthold, M.R.; Cebron, N.; Dill, F.; Gabriel, T.R.; Kötter, T.; Meinl, T.; Ohl, P.; Thiel, K.; Wiswedel, B. KNIMEthe Konstanz information miner: version 2.0 and beyond. AcM SIGKDD Explor. Newsl. 2009, 11, 26-31.

19. Rojas Vargas, J.A.; López, A.G.; Pérez, Y.; Cos, P.; Froeyen, M. In vitro evaluation of arylsubstituted imidazoles derivatives as antiprotozoal agents and docking studies on sterol 14 $\alpha$-demethylase (CYP51) from Trypanosoma cruzi, Leishmania infantum, and Trypanosoma brucei. Parasitol. Res. 2019, 1533-1548.

20. RCSB Protein Data Bank.

21. CLC Bio Company Mollegro Virtual Docker 6.0.

22. Barros, R.P.C. Triagem virtual de metabólitos secundários com potencial atividade antimicrobiana do gênero solanum e estudo fitoquimico de solanum Capsicoides all. 2017.

23. Acevedo, C.H.; Scotti, L.; Scotti, M.T. In Silico Studies Designed to Select Sesquiterpene Lactones with Potential Antichagasic Activity from an In-House Asteraceae Database. ChemMedChem 2018, 13, 634-645. 